\title{
Proyecto pedagógico para disminuir el consumo del biberón en la primera infancia*
}

\author{
Pedagogical Project to Reduce Feeding Bottle Consumption in Early Childhood \\ Laura Lobón ${ }^{1}$ Dayanna Lema² Yency Quintero ${ }^{3}$ Melissa Sánchez ${ }^{4}$
}

Para citar este artículo: : Lobón, L., Lema, D., Quintero, Y., Sánchez, M. (2018). Proyecto pedagógico para disminuir el consumo del biberón en la primera infancia. Infancias Imágenes, 17(1), 53-66.

\section{Resumen}

El desarrollo de la presente investigación se dio para dar respuesta a la pregunta ipuede un proyecto pedagógico, disminuir el consumo del biberón en niños de 2 a 5 años de edad en dos hogares del ICBF Pereira? Se tomaron en cuenta referentes teóricos como: hábito del biberón, control de hábitos, educación para la salud, promoción de la salud y pedagogía por proyectos. Se aplicó una metodología enfocada en la pedagogía por proyectos, apoyada en talleres pedagógicos basados en los conocimientos previos de la comunidad. Como resultado se obtuvo que la mitad de la población, compuesta por 14 niñas y niños, abandonó el hábito del biberón y la otra mitad disminuyó la frecuencia del consumo; con tutores y agentes educativas se logró aumentar el conocimiento sobre el hábito. Se concluyó que la pedagogía por proyectos y la educación para la salud son herramientas clave para lograr cambios positivos en comunidades.

Palabras clave: primera infancia; educación de los padres; educación para la salud; hábito; biberón; salud bucal.
Recibido: 05-diciembre-2017 / Aprobado: 14-febrero-2018

\section{Abstract}

The development of this research was given to answer the question: Can a pedagogical project reduce consumption of feeding bottle in children from 2 to 5 years of age in two homes of Pereira ICBF? Theoretical referents were taken into account, such as: bottle-feeding habit, habit control, health education, health promotion and pedagogy by projects. A methodology focused on pedagogy by projects was applied, supported by pedagogical workshops based on the community's prior knowledge. As a result, it was obtained that half of the population, composed of 14 girls and boys, abandoned bottle-feeding habit and the other half decreased the consumption frequency; it was possible to increase knowledge about the habit with tutors and educational agents. We conclude that pedagogy by projects and health education are key tools to achieve positive changes in communities.

Keywords: early childhood; parental education; health education; habit; feeding bottle; oral health.

\footnotetext{
* Artículo que presenta los resultados de la investigación "Pedagogía por proyectos para disminuir el consumo del biberón en niñas y niños de 2 a 5 años incluyendo a sus respectivos tutores en los hogares del ICBF Mimosín y los Pollitos, Pereira Risaralda en el 2017“. Esta inició en septiembre de 2016 y finalizó en julio de 2017. Avalada por la Fundación Universitaria Autónoma de las Américas (sede Pereira).

1 Odontóloga, Fundación Universitaria Autónoma de las Américas. Correo electrónico: laura.lobon@uam.edu.co

2 Odontóloga, Fundación Universitaria Autónoma de las Américas. Correo electrónico: dayana.lema@uam.edu.co

3 Odontóloga, Fundación Universitaria Autónoma de las Américas. Correo electrónico: yency.quintero@uam.edu.co

${ }^{4}$ Odontóloga, Fundación Universitaria Autónoma de las Américas, especialista en Gerencia de la Calidad y Auditoria en Salud, Universidad Cooperativa de Colombia. Profesora Facultad de Odontología e investigadora del Grupo de Investigación en Salud y Comunidad de la Fundación Universitaria Autónoma de las Américas, Pereira, Colombia. Correo electrónico: melissa.sanchez@uam.edu.co
} 


\section{Introducción}

La salud bucodental es un componente de la salud general de los seres humanos y es vital para el adecuado crecimiento y desarrollo de niñas y niños (Castro, 2017). Sin embargo, las enfermedades bucodentales constituyen un importante problema debido a su alta tasa de prevalencia, demanda en las consultas dentales y fuerte impacto en la sociedad en términos de dolor, malestar, limitación y discapacidad social y funcional, así como también por su efecto sobre la calidad de vida en la población (Tello, Abanto, Butini, Murakami, Bonini y Bönecker, 2016). Entre las patologías bucodentales más frecuentes, especialmente en los infantes, se encuentra la caries dental, la cual está altamente asociada al consumo del biberón y toma el nombre de caries de la infancia temprana (CIT). Si bien este es un complemento alimenticio hasta los 2 años, después se convierte en un hábito nocivo que ocasiona alteraciones tales como: prognatismo maxilar, retrognatia mandibular, incisivos superiores vestibularizados y paladar ojival. Estas alteraciones, a largo plazo, necesitan tratamientos ortodónticos, ortopédicos, quirúrgicos, entre otros, que afectan la salud en general (Salamanca y Murrieta, 2015).

En términos generales, la prevalencia de la caries varía dependiendo de los diferentes determinantes sociales como las condiciones socioeconómicas, región geográfica, disponibilidad y accesibilidad a los servicios de salud bucodental, ocupación y el grado de educación (García, Sánchez y Estrada, 2015). La CIT tiene una prevalencia de $12 \%$ en los países desarrollados y de $70 \%$ en los países en vías de desarrollo (Torres, Blanco, Chávez, Apaza y Antezana, 2015). Asimismo, el Estudio nacional de salud bucal III (Ensab III) (Ministerio de Salud, 1999) determinó que la prevalencia de caries en dentición temporal fue de 45,7\%. Si esta cifra se compara con el siguiente estudio, es decir, el Ensab IV (Ministerio de Salud y Protección Social, 2014), se nota que aumentó a un $62,24 \%$.

En el plano local, mientras que la Secretaría de Salud y Seguridad Social de Pereira (2014) reportó que las enfermedades bucodentales se encuentran entre las principales causas de morbilidad desde el primer año de vida, la Secretaría de Salud de Risaralda (2017) reveló que en Pereira la CIT fue la primera causa de morbilidad por consulta externa con 62.924 casos. Sin embargo, a pesar de que se conoce que el hábito del biberón es el principal factor de riesgo para desarrollar tal patología, no se reportan investigaciones en la ciudad sobre este.

De lo anterior se desprende que la atención integral de la primera infancia es un tema de política pública en Colombia. En este sentido, el Instituto Colombiano de Bienestar Familiar (ICBF, 2014), con el programa de Hogares Comunitarios, atiende las necesidades básicas de protección, cuidado, nutrición, salud, educación y desarrollo psicosocial de los niños y niñas. Esta iniciativa se realiza en las viviendas de las agentes educativas o madres comunitarias, previamente capacitadas, quienes se responsabilizan por el cuidado y atención de grupos conformados por entre 12 a 14 niños y niñas durante ocho horas diarias; además, deben mantener comunicación constante con los tutores de los menores.

En tal sentido, se debe vincular a los responsables de la primera infancia para mejorar las condiciones de salud. Al respecto, Gómez, Bernal, Posada y Agudelo (2015) evidenciaron la relación entre la falta de conocimiento sobre el cuidado bucal que tienen los tutores de las niñas y los niños con la deficiencia en la higiene bucal y el consumo del biberón. Además, en una investigación realizada por González, Cano, Meneses y Saldarriaga (2016), las agentes educativas reconocieron ser las responsables de la higiene oral de los niños mientras estuvieran en el hogar comunitario; sin embargo, no cuentan con los conocimientos ni la destreza adecuada para cepillarlos, ya que los profesionales de la salud no se toman el tiempo de explicarles cómo hacerlo, sumado a esto los padres de familia no complementan dicha tarea en el hogar. Por lo anterior, mediante la pedagogía por proyectos (PP) y la educación para la salud se buscó enseñar a los participantes de la investigación las consecuencias que trae el uso prolongado del biberón.

Autores como Jiménez, Galvis, Gómez, Salinas y Agudelo (2015) dan cuenta de que al utilizar la metodología de PP se logran resultados en salud, ya que parte de las necesidades o conocimientos previos de la comunidad se construyen y afianzan a medida que avanza la investigación, creando así un 
aprendizaje significativo en los participantes. Además, se involucra a todos los actores: comunidad e investigadores mediante un acompañamiento continuo que hace efectiva la intervención promoviendo la salud de los participantes, específicamente de la primera infancia.

En dicho contexto es importante emprender acciones preventivas que se conviertan en estrategias para la promoción de la salud, que lleven a la comunidad a un cambio de conductas a través de la construcción y el afianzamiento de conocimientos, que sean útiles para el sano vivir (Contreras, 2016; De Luca, Rivera y Romero, 2015).

En este orden de ideas, el objetivo de la presente investigación fue desarrollar un proyecto pedagógico en dos hogares comunitarios del ICBF en la ciudad de Pereira para disminuir el consumo del biberón en las niñas y los niños de 2 a 5 años de edad en la que se incluyen a sus respectivos tutores en el periodo 2017-I.

\section{Marco teórico}

Para esta investigación fue necesario tomar en cuenta conceptos tales como: hábito del biberón, alteraciones provocadas por el hábito del biberón, control de hábitos, educación para la salud, promoción de la salud y PP.

\section{Hábito del biberón}

Los hábitos orales hacen referencia a prácticas que se adquieren a partir de la constante realización. Se aprenden en los primeros meses a partir de la enseñanza de los padres, quienes, en este caso, emplean el uso del biberón en reemplazo del seno de la madre como una forma alterna de alimentación. Después de ser aprehendido su realización es inconsciente (Myers, 2005).

El hábito del biberón comienza cuando se refuerza la alimentación del niño, pero si se exagera su uso (en mayores de 2 años) se convierte en un hábito potencialmente dañino para el infante, ya que puede afectar su entorno social, emocional, funcional y psicológico. Según el psicólogo Harry Harlow (citado por Myers, 2005), después de su nacimiento, los infantes empiezan a tener ciertas conductas como la succión, sonrisas reflejas, el balbuceo, la necesidad de ser arrullado y el Ilanto, lo cual crea un vínculo con sus padres y, asimismo, desarrollan apego hacia ellos. Así, cuando los padres se alejan se genera en los bebés "ansiedad de separación", momento en el cual el biberón reconforta dicha ansiedad porque se convierte en una base segura para alivianar momentos de estrés y, además de ello, con él exploran el mundo por medio de la boca; por estas razones es tan difícil para los niños deshacerse de este hábito. Adicionalmente, cuando la succión del biberón se prolonga más allá del segundo año de vida conlleva a alteraciones a nivel oro-facial; en otros casos también puede producir alteraciones en la correcta oclusión dentaria (Nihi et al., 2015), lo que trae consigo la afectación de la salud oral de los niños.

Por tanto, es necesario generar estrategias y acciones que contribuyan en la enseñanza y promoción de nuevas prácticas para promover hábitos adecuados en los cuidadores con respecto a la alimentación y el límite indicado para el retiro del biberón. Como se ha visto, su uso constante, repetitivo y prolongado en el tiempo puede causar múltiples alteraciones como las que se mencionarán a continuación.

Alteraciones provocadas por el hábito del biberón El uso prolongado de biberón ha sido relacionado con alteraciones en el crecimiento y desarrollo de los maxilares. Al respecto, Gil afirma que:

Con el uso de biberón el niño no cierra los labios con tanta fuerza y éstos adoptan forma de ' $\mathrm{O}$ ', no se produce el vacío bucal, se dificulta la acción de la lengua, la cual se mueve hacia adelante contra la encía para regular el flujo excesivo de leche, se mantiene plana hay menor excitación a nivel de la musculatura bucal que tenderá a convertirse en hipotónica y no favorecerá el crecimiento armonioso de los huesos. (2017, p. 10)

Cuando los infantes se alimentan por medio del seno materno realizan más esfuerzo al succionar, lo cual es beneficioso para el adecuado desarrollo fisiológico, anatómico y funcional de las estructuras maxilofaciales. Por el contrario, al utilizar el biberón el niño realiza un esfuerzo mínimo para obtener 
su contenido, lo que producirá una modificación en la anatomía de los arcos, erupción y ubicación de los dientes además de relaciones maxilo-mandibulares inadecuadas que se verán reflejadas en maloclusiones (Gil, 2017; Chen, Xia y Ge, 2015). De esta forma se presentan las siguientes alteraciones provocadas por el hábito del biberón:

- La CIT: definida como una forma severa y particular de caries, de carácter multifactorial, que afecta la dentición temporal de lactantes y niños pre-escolares comprometiendo, por lo general, numerosos dientes y genera la rápida destrucción e infección subsiguiente del tejido dentario. Está asociada con la ingesta excesiva de cualquier líquido azucarado contenido en el biberón como la leche, fórmulas, jugos de frutas, refrescos y la falta de higiene después de la ingesta (Aguilar, Duarte, Rejón, Serrano y Pinzón, 2014).

- Paladar ojival: más conocido como paladar profundo, se caracteriza por ser cóncavo con forma ovalada, adoptando la forma de la tetina del biberón que el niño o niña consume (Ocampo, Johnson y Lema, 2014).

- Retrognatia mandibular: se ocasiona por la falta de desarrollo mandibular en relación al maxilar o por el exceso de desarrollo del maxilar en relación con la mandíbula. Esta alteración se reporta en los pacientes que consumen o consumieron biberón en su infancia afectando su oclusión dentaria (Reyes, Torres, Quesada, Milá y Labrada, 2014).

- Mordida abierta anterior: se puede definir como la ausencia de contacto de los dientes pertenecientes a los sectores anteriores de los maxilares con sus antagonistas. El biberón se interpone entre los dientes e impide el contacto normal de estos (Reni y Piñeiro, 2014).

- Mordida cruzada posterior: "los segmentos posteriores (premolares y molares) del maxilar superior se encuentran en posición lingual con respecto a sus homólogos de la mandíbula" (Universidad Nacional de Colombia, 2016, p. 8).

- Oclusión clase II división I (distoclusión): cuando el primer molar superior ocluye hacia distal del primer molar inferior, es división I si los incisivos superiores están vestibularizados, esta posición se ocasiona por el consumo del biberón (Reni y Piñeiro, 2014).

- La deglución atípica: alteración provocada por el hábito del biberón, ya que los movimientos de la lengua son inadecuados y se afecta la fase bucal y faringolaríngea de la deglución (Jiménez, 2016).

Estas alteraciones son prevenibles si se disminuye y elimina el hábito de biberón a los 2 años de vida, lo que resalta la importancia de realizar intervenciones de educación para la salud que se concentren en disminuir y/o eliminar esta práctica, evitando a futuro la necesidad de tratamientos ortodónticos, ortopédicos y quirúrgicos.

\section{Control de hábitos}

Según Acevedo, Mora, Capote, Rosell y Hernández (2011), el primer paso para tratar un hábito nocivo es descubrir su agente causal, ya que al ser detectado y adecuadamente tratado puede conllevar a la desaparición de este. Asimismo, en una investigación realizada por este autor, se resalta la efectividad de la educación para eliminar por completo algunos hábitos, entre estos el uso del biberón, cuyo resultado evidenció la disminución de $58,2 \%$ a $11,9 \%$ en niños que hacían uso de este. Generalmente, los hábitos comienzan en edades tempranas, lo que hace más difícil su eliminación porque los niños aún no comprenden sus posibles consecuencias.

Por lo que ésta es la tarea más importante y en ocasiones compleja al tratar de convencer al paciente del daño que ocasiona la práctica de cualquiera de los hábitos; es importante, además, la cooperación de los padres pues la llave para la eliminación satisfactoria de un hábito es la motivación de padre e hijo. (Corrales, Duque, Serrano, González y Alonso, 2014, p. 1002)

Educación para la salud y promoción de la salud La educación para salud es un elemento clave para prevenir, curar y rehabilitar diferentes enfermedades y se convierte en instrumento de 
la promoción de la salud (Peñaranda, López y Molina, 2017). En esta, las personas son empoderadas para que puedan adoptar medidas encaminadas al mejoramiento de su salud y de la comunidad, tomando en cuenta los factores y comportamientos de riesgo, uso de los servicios de salud y las condiciones sociales, ambientales y económicas que pueden tener influencia en esta (Unesco, 1999).

En cuanto a la educación en salud bucal, Contreras (2016) la define "como una combinación de oportunidades y aprendizajes que facilita cambios voluntarios del comportamiento y que conducen a una mejora de la salud bucal, teniendo en cuenta que la salud dental es inseparable de la salud en general“ (p. 195).

La educación para la salud no solo se basa en la transmisión de información a las personas, sino que radica en motivarlas para que tengan actitud positiva en sus comportamientos $y$, finalmente, persuadirlas para que desarrollen capacidades y puedan tomar decisiones libres y autónomas que favorezcan positivamente su situación de salud (Carlos, Luengo, Toscano, Luévano y Anaya, 2017). Según la Unesco:

La educación para la salud puede interpretarse como un elemento de promoción de la salud. La promoción de la salud y la educación para la salud se complementan y tienen como propósito mejorar el acceso a la información y a los servicios relacionados con la salud, con el fin de que la gente obtenga un mayor control sobre su propia salud y su propio bienestar" (1999, p.7)

Pedagogía por proyectos

Rincón define la PP como:

Una estrategia que busca la eficiencia y eficacia en los aprendizajes de una persona, involucrando a maestros y alumnos creando en ellos un aprendizaje significativo, esta se apoya en diferentes categorías, tales como, la enseñanza y el aprendizaje, estableciendo vínculos entre los saberes con los que cuenta una persona y los nuevos que pueda adquirir, a través de procesos de discusión, interacción, confrontación, documentación, y por último, la construcción de significados. (2012, p. 80)

Esta a su vez se desarrolla en cuatro fases:

Exploración: se identifica el tema a ser desarrollado.

Planeación: se busca responder a preguntas como: ¿qué se va a hacer o aprender (tema o problema)?, sobre ese tema o problema ¿qué se quiere aprender?, ¿para qué?, ¿por qué?, ¿cómo?, ¿cuándo?, ¿dónde?, ¿con quiénes, o con qué?, ¿cómo se va a llamar el proyecto?, ¿cómo se va a evaluar el proyecto?

Desarrollo: es la ejecución del proyecto de acuerdo a la planeación para lograr los objetivos propuestos.

Evaluación/socialización: para revisar los aprendizajes logrados con la realización del proyecto y compartirlo con otras personas.

La PP se orienta, desde algunos principios, como la participación en las actividades, las formas de regulación, las formas del discurso, la cooperación con otros y la primacía en los intereses del niño (Rincón, 2012).

En conclusión, la PP parte de los problemas de la vida diaria y rompe los esquemas de la educación tradicional permitiendo a aquellas personas que forman parte de él participar desde planeación hasta la ejecución. Así pues, esta propuesta pedagógica permite plantear la construcción de planes en conjunto con agentes comunitarias, padres de familias, niñas y niños, diseñando actividades en las que se toma de antemano lo que se desea mejorar de la vida diaria mediante un acompañamiento continuo que hace que sea efectiva la intervención (Jiménez et al., 2015).

\section{Metodología}

Se escogió para esta investigación un enfoque cualitativo y como método la PP, con la que se buscó que los participantes fueran protagonistas de su propio cambio. La población de estudio estuvo conformada por 14 niñas y niños entre los 2 y 5 años que consumían biberón y asistían a los hogares comunitarios Mimosín y Los Pollitos del ICBF 
del barrio Cuba, en Pereira, Risaralda. De la misma manera, fueron abordados los 14 tutores legales de los niños que correspondían a sus padres de familia y dos agentes educativas, quienes fueron incluidos en el proyecto una vez firmaron los consentimientos informados.

Se realizaron seis sesiones con las niñas y niños y cinco con los padres y agentes educativas; todas con una intensidad de una hora y 30 minutos en un lapso de tres meses durante el primer semestre del 2017. Como técnica de investigación se utilizó el taller pedagógico — considerado como un proceso consciente, deliberado y participativo implementado con la comunidad participante- para fortalecer el conocimiento en cuanto al cuidado bucal de la primera infancia y disminuir concretamente el consumo de biberón.

Como herramientas de recolección de datos se utilizaron diarios de campo. Además, se dividió la población en un grupo focal inicial y uno final; en ambos los participantes fueron codificados, para así realizar el análisis de la información. Por ejemplo: 1G1, el primer número corresponde al número asignado a la persona, G significa grupo focal y el segundo número relaciona si es el grupo focal inicial 1 o final 2.

Este proyecto pedagógico se realizó mediante las siguientes cuatro fases: exploración, planeación, ejecución y evaluación.

En la fase de exploración se indagaron los conocimientos, inquietudes, sentimientos, opiniones y experiencias previas acerca del consumo del biberón y sus posibles consecuencias. A los niños se les proyectó un video alusivo a dicho hábito y fueron cuestionados si lo consumían o no. Con los tutores y agentes educativas se conformó un grupo focal inicial en el que se abordaron preguntas relacionadas con la salud bucal y el hábito del biberón, tales como: ¿hasta qué edad se debe consumir biberón?, ¿qué es la caries?, ¿con qué frecuencia consume su hijo biberón?, ¿qué consecuencias trae el hábito del biberón?, ¿creen que el tetero es bueno o malo y por qué?, en las prácticas diarias de higiene oral ¿sus hijos se cepillan solos o ustedes les ayudan?; además de preguntar por algunos datos sociodemográficos.
En la planeación, a partir del grupo focal inicial, se diseñaron con los padres y agentes educativas las actividades y temas a tratar con ellos. Para saber las preguntas que tenían los niños y niñas sobre el biberón y la cavidad bucal, se utilizó un macro modelo y un biberón como material estimulante de inquietudes, además se planearon las acciones para responder a dichos interrogantes, de acuerdo con la capacidad de entendimiento de la primera infancia.

En la fase de ejecución se implementaron las actividades diseñadas con niños, niñas, agentes educativas y padres de familia. Estas se muestran en el apartado de resultados, ya que fueron construcciones realizadas con la comunidad participante.

En la última fase, la de evaluación, se conformó un grupo focal final con los tutores y agentes educativas con las mismas preguntas del grupo focal inicial, con el fin de contrastar las respuestas iniciales y las finales. Con los niños se realizó una actividad evaluativa la cual consistió en enterrar el tetero con una previa explicación de las razones por las que lo debían hacer.

Para el análisis de la información se utilizaron los principios de la teoría fundamentada. Se agruparon los datos para su respectivo análisis y se realizó la codificación abierta en la cual, por comparación constante de los datos, emergieron diferentes categorías de análisis. De acuerdo con esto, se llevó a cabo la codificación axial de la que emergió la categoría central hábito del biberón, esta se relacionó con las demás categorías y se comparó las respuestas iniciales y finales de los participantes.

En la investigación se utilizó un formato de asentimiento informado para la participación de la primera infancia, y otro de consentimiento informado para los tutores y agentes educativas. Esta investigación tiene el aval del Comité de Ética y Bioética de la Fundación Universitaria Autónoma de las Américas. Y de acuerdo con la Resolución 8430 de 1993 de Colombia, la investigación se clasifica como de riesgo mínimo.

\section{Resultados y discusión}

La población participante estuvo conformada por 14 niñas y niños, 14 tutores legales responsables de la primera infancia y dos agentes educativas. Sus respectivos datos sociodemográficos se reportan en la tabla 1. 
Tabla 1. Datos sociodemográficos de los participantes en la investigación.

\begin{tabular}{|l|l|l|}
\hline \multicolumn{1}{|c|}{ Variable } & \multicolumn{1}{|c|}{ Primera infancia } & \multicolumn{1}{c|}{ Tutores y agentes educativas } \\
\hline Estrato socioeconómico & $1(23 \%) 2(45 \%) 3(32 \%)$ & $1(23 \%) 2(45 \%) 3(32 \%)$ \\
\hline \multirow{3}{*}{ Edad } & $\begin{array}{l}2 \text { años: }(21 \%) \\
3 \text { años: }(15 \%) \\
4 \text { años: }(43 \%) \\
5 \text { años: }(21 \%)\end{array}$ & $\begin{array}{l}\text { Entre 20-30 años: }(57 \%) \\
\text { Entre 30-40 años: }(29 \%) \\
\text { Entre } 40-50 \text { años: }(14 \%)\end{array}$ \\
\hline Hombres & $(57 \%)$ & $(21 \%)$ \\
\hline Mujeres & $(43 \%)$ & $(79 \%)$ \\
\hline \multirow{3}{*}{ Nivel educativo } & $\begin{array}{l}\text { Programa de cero a siempre } \\
(100 \%)\end{array}$ & $\begin{array}{l}\text { Primaria: }(7 \%) \\
\text { Secundaria: }(14 \%) \\
\text { Bachiller: }(50 \%) \\
\text { Técnico: }(29 \%)\end{array}$ \\
\hline
\end{tabular}

Fuente: elaboración propia de las autoras.

Las diferentes actividades realizadas en cada fase de la PP durante el desarrollo de esta investigación buscaron fomentar la participación de los niños, padres y agentes educativas para disminuir el hábito del biberón, teniendo en cuenta sus saberes previos e inquietudes con el objeto de construir en pro de la salud bucal de la primera infancia. Sánchez, Sánchez y Agudelo (2015) afirman que los proyectos se deben orientar hacia la búsqueda de una participación activa, el uso del lenguaje como herramienta fundamental para establecer relaciones con las demás personas y construir conocimientos.

En la fase de exploración se realizaron los primeros acercamientos a la comunidad con el desarrollo de actividades destinadas a romper el hielo y a crear vínculos de confianza entre los participantes y las investigadoras; permitiéndoles expresarse de forma abierta, sin sentirse intimidados u obligados a participar. Esto concuerda con la propuesta de Varela y Vives (2016), quienes dan cuenta de los proyectos de enfoque cualitativo en la medida en que buscan reconstruir la realidad tal cual se observa y pretenden comprender el contexto que rodea a los participantes, en virtud de la exploración e interpretación de las acciones de las personas creando vínculos de confianza con la población investigada.

De este modo, se recolectó información a través del grupo focal inicial en el que los padres y las agentes educativas refirieron que las niñas y los niños consumían biberón tres o cuatro veces al día desde hacía más de dos años. Además, revelaron en sus respuestas conocimientos erróneos acerca de la edad límite para consumir el biberón y las consecuencias que esto trae; tampoco tenían certeza sobre si el hábito del biberón era o no saludable y ni dieron cuenta de lo que realmente es la caries, tal como se evidencia en los siguientes fragmentos:

"Yo me siento segura dándole tetero a mi hija" (1G1)

"El tetero es bueno porque quedan más llenitos y se duermen más fácil" (2G1)

"Yo digo que el teterito se debe dejar a los 4 años" (4G1)

"La caries da por comer dulces" (3G1)

Al respecto, González, Pérez, Alarcón y Peñalver (2015) evidencian que los padres de los niños que tienen el hábito prolongado del biberón presentan deficiencias en el nivel de conocimiento acerca de la salud oral de sus hijos. Esto se explica por la falta de efectividad en los diferentes métodos de promoción de la salud.

Con los niños se recolectó información después de mostrarles un video alusivo al biberón, al cuestionárseles por el consumo todos respondieron que les gustaba. También se llevó a cabo la actividad "Atrapa la pelota" con el objetivo de generar lazos de confianza; en esta se conocieron sus nombres, sus gustos y frecuencia del consumo de biberón, así se realizó el primer acercamiento con ellos. 
En la fase de planeación, al cuestionar a los tutores y agentes educativas sobre cómo querían aprender ellos y sus hijos, la mayoría de padres coincidió en que los niños lo harían con actividades lúdicas, mientras que los adultos tendrían mejor aprendizaje a través de presentaciones, conferencias, ayudas visuales, entre otras. En efecto, con los niños y niñas se trabajó con un macro modelo dental y con un biberón, mientras ellos hacían preguntas acerca de los dientes y los tejidos blandos de la cavidad oral. Como lo declara Rincón (2012), la PP se construye a partir de las necesidades y conocimientos previos de las personas. De esta manera, se diseñaron las actividades tanto para los niños como para los padres.
Actividades con los niños

La fase de ejecución se realizó de manera independiente, una con los niños y otra con los padres y las agentes educativas. En la tabla 2 se evidencian las actividades con los niños y niñas.

"Los súper odontólogos" fue la primera actividad de la fase de ejecución, esta pretendió el aprendizaje de las partes de la cavidad oral y sus respectivas funciones. Se disfrazó a cada niño con capas de colores, gorros, tapabocas, guantes y se examinaron entre ellos; reconocieron así estructuras de la boca como la lengua, los dientes, el paladar, las amígdalas, las encías, entre otras, logrando una conjugación entre el aprendizaje y la diversión como lo relacionan Jiménez et al. (2015) en su estudio.

Tabla 2. Actividades con primera infancia.

\begin{tabular}{|c|c|c|c|}
\hline Fase & Actividades con los niños & Material utilizado & $\begin{array}{l}\text { Tiempo de } \\
\text { duración }\end{array}$ \\
\hline $\begin{array}{l}\text { Fase de } \\
\text { exploración }\end{array}$ & $\begin{array}{l}\text { Presentación de video alusivo al biberón y después } \\
\text { de este se pregunta a los niños si lo consumen o no. } \\
\text { "Atrapa la pelota", actividad en la cual se rompe } \\
\text { el hielo con las niñas y niños para promover la } \\
\text { participación en las actividades. }\end{array}$ & $\begin{array}{l}\text { Pelota, preguntas } \\
\text { sobre frecuencia, } \\
\text { duración e intensidad } \\
\text { del consumo del } \\
\text { biberón, y video } \\
\text { beam. }\end{array}$ & 90 minutos \\
\hline $\begin{array}{l}\text { Fase de } \\
\text { planeación }\end{array}$ & $\begin{array}{l}\text { Se utilizó un macro modelo y un biberón como } \\
\text { material estimulante de preguntas para conocer } \\
\text { las inquietudes que tenían los niños y niñas sobre } \\
\text { el biberón y la cavidad bucal. Posteriormente, } \\
\text { se diseñaron las actividades de acuerdo con la } \\
\text { capacidad de entendimiento de la primera infancia. }\end{array}$ & $\begin{array}{l}\text { Computador, papel, } \\
\text { macro modelo y } \\
\text { biberón. }\end{array}$ & 120 minutos \\
\hline \multirow{3}{*}{$\begin{array}{l}\text { Fase de } \\
\text { ejecución }\end{array}$} & $\begin{array}{l}\text { "Los súper odontólogos", actividad en la cual las } \\
\text { niñas y niños identificaron partes de la cavidad oral } \\
\text { (la lengua, los dientes, el paladar, las amígdalas, las } \\
\text { encías) y sus respectivas funciones. }\end{array}$ & $\begin{array}{l}\text { Capas de colores, } \\
\text { gorros, tapabocas y } \\
\text { guantes. }\end{array}$ & 90 minutos \\
\hline & $\begin{array}{l}\text { "Los monstruos del tetero", obra de teatro para } \\
\text { persuadir a los niños sobre las consecuencias } \\
\text { negativas que puede causar el consumo prolongado } \\
\text { del biberón como caries y maloclusiones. }\end{array}$ & $\begin{array}{l}\text { Tetero gigante, } \\
\text { insectos de juguete, } \\
\text { muñeca de trapo y } \\
\text { obra de teatro. }\end{array}$ & 90 minutos \\
\hline & $\begin{array}{l}\text { "Revelando la placa", en la cual se les aplicó gotas } \\
\text { reveladoras de placa bacteriana a cada uno de los } \\
\text { niños y se asoció la pigmentación rosada de los } \\
\text { dientes con los "monstruos del tetero". Después } \\
\text { de la aplicación de las gotas se continuó con la } \\
\text { actividad de cepillado colectivo en la cual se les } \\
\text { mostró cómo debían cepillarse y usar el hilo dental } \\
\text { para eliminar dichos "monstruos" y con esto se les } \\
\text { enseñó prácticas saludables en higiene oral. }\end{array}$ & $\begin{array}{l}\text { Gotas reveladoras y } \\
\text { kit de cepillado. }\end{array}$ & 90 minutos \\
\hline $\begin{array}{l}\text { Fase } \\
\text { evaluación }\end{array}$ & $\begin{array}{l}\text { "Enterrando recuerdos", actividad en la cual cada } \\
\text { niño, por iniciativa propia, decide enterrar el tetero y } \\
\text { explica las razones por las cuales lo debe abandonar. }\end{array}$ & $\begin{array}{l}\text { Teteros, palas, tierra } \\
\text { y vasos. }\end{array}$ & 90 minutos \\
\hline
\end{tabular}


En la siguiente visita se realizó una actividad Ilamada "Los monstruos del tetero", basada en una obra de teatro para persuadir a los niños sobre las consecuencias negativas que puede causar el consumo prolongado del biberón como caries y maloclusiones, lo que condujo a poner a prueba su imaginación e intentar así la construcción de aprendizaje significativo. Esta actividad está en sintonía con la experiencia descrita por Jiménez et al. (2015) en la cual se utilizaron juguetes como una muñeca, un biberón y diferentes insectos llamados "monstruos del tetero", los cuales eran la representación gráfica de la caries y las bacterias de la cavidad oral.

En otra visita se desarrolló la actividad "Revelando la placa" que consistió en la aplicación de gotas reveladoras de placa bacteriana a cada uno de los niños y se asoció la pigmentación rosada de los dientes con los "monstruos del tetero". Después se continuó con una actividad de cepillado colectivo, en la que se indicó cómo debían cepillarse y usar el hilo dental para eliminar dichos monstruos; con esto se les enseñó prácticas saludables en higiene oral. Lo anterior estuvo sustentado en los planteamientos de Ávila, Leiva, Bueno y Gámez (2016), quienes coinciden en que la primera infancia es la etapa del ciclo vital más adecuado para potenciar las capacidades cognitivas, comunicativas y sociales de los niños enfocadas a la salud oral.

Actividades con los padres y agentes educativas En la fase de ejecución con los padres y las agentes educativas se realizaron varias actividades evidenciadas en la tabla 3.

Las actividades con los padres se diseñaron de acuerdo con las falencias evidenciadas en el grupo focal inicial acerca del hábito del biberón. En estas se utilizaron ayudas visuales y gráficas como la proyección en video beam, presentaciones en PowerPoint y un macro modelo que simulaba la cavidad oral.

Tabla 3. Actividades con los padres y agentes educativas.

\begin{tabular}{|c|c|c|c|}
\hline Fase & Actividades con los tutores & Materiales & Duración \\
\hline $\begin{array}{l}\text { Fase de } \\
\text { exploración }\end{array}$ & $\begin{array}{l}\text { Grupo focal inicial se realizaron preguntas relacionadas } \\
\text { con la salud bucal y el hábito del biberón para conocer } \\
\text { inquietudes, sentimientos, opiniones y experiencias sobre } \\
\text { este. Se indagó de qué manera querían aprender ellos } \\
\text { y sus hijos y, además, se preguntó por algunos datos } \\
\text { sociodemográficos. }\end{array}$ & $\begin{array}{l}\text { Papel, grabadora de } \\
\text { audio y lapicero }\end{array}$ & $\begin{array}{l}90 \\
\text { minutos }\end{array}$ \\
\hline $\begin{array}{l}\text { Fase de } \\
\text { planeación }\end{array}$ & $\begin{array}{l}\text { Se diseñaron las actividades y temas a tratar con ellos de } \\
\text { acuerdo con lo encontrado en el grupo focal inicial. }\end{array}$ & $\begin{array}{l}\text { Papel, grabadora de } \\
\text { audio y computador. }\end{array}$ & $\begin{array}{l}120 \\
\text { minutos }\end{array}$ \\
\hline \multirow{3}{*}{$\begin{array}{l}\text { Fase de } \\
\text { ejecución }\end{array}$} & $\begin{array}{l}\text { Importancia de los dientes temporales: se explica a los padres } \\
\text { y agentes educativas la necesidad de cuidar los dientes } \\
\text { temporales para la adecuada erupción de los permanentes. }\end{array}$ & $\begin{array}{l}\text { Video beam y } \\
\text { PowerPoint. }\end{array}$ & $\begin{array}{l}90 \\
\text { minutos }\end{array}$ \\
\hline & $\begin{array}{l}\text { Importancia de abandonar el hábito del biberón: se explica } \\
\text { a los tutores y agentes educativas las repercusiones negativas } \\
\text { que trae dicho hábito para sus hijos y el beneficio que } \\
\text { conllevaría el abandonarlo tanto a nivel funcional de la } \\
\text { cavidad oral como a nivel emocional. }\end{array}$ & $\begin{array}{l}\text { Video beam, } \\
\text { macromodelo } \\
\text { gigante cavidad oral } \\
\text { y PowerPoint. }\end{array}$ & $\begin{array}{l}90 \\
\text { minutos }\end{array}$ \\
\hline & $\begin{array}{l}\text { Consecuencias del hábito del biberón: actividad que muestra } \\
\text { a los tutores y agentes educativas las secuelas negativas } \\
\text { del hábito del biberón, entre las cuales se encuentran } \\
\text { maloclusiones, caries de la infancia temprana, alteraciones } \\
\text { faciales, oclusales, del lenguaje y del desarrollo físico y } \\
\text { emocional del niño que terminarán, si no se tratan a tiempo, } \\
\text { en costosos tratamientos terapéuticos y correctivos. }\end{array}$ & $\begin{array}{l}\text { Video beam y } \\
\text { PowerPoint }\end{array}$ & $\begin{array}{l}90 \\
\text { minutos }\end{array}$ \\
\hline $\begin{array}{l}\text { Fase de } \\
\text { evaluación }\end{array}$ & $\begin{array}{l}\text { Grupo focal final, con las mismas preguntas del inicial, } \\
\text { contrastando las respuestas iniciales y finales. }\end{array}$ & $\begin{array}{l}\text { Papel, grabadora de } \\
\text { audio y lapicero. }\end{array}$ & $\begin{array}{l}90 \\
\text { minutos. }\end{array}$ \\
\hline
\end{tabular}

Fuente: elaboración propia de las autoras. 
En el primer taller pedagógico se abordaron temas como la importancia de los dientes temporales para la adecuada erupción de los dientes permanentes, la edad de erupción de los dientes permanentes y la importancia de dejar el biberón para evitar futuros tratamientos de ortopedia y ortodoncia como demuestran Salamanca y Murrieta (2015). Estos últimos argumentan que la presencia de hábitos deformantes como el biberón trae consigo la aparición de alteraciones faciales, oclusales, del lenguaje y del desarrollo físico y emocional del niño que terminarán en costosos tratamientos terapéuticos y correctivos si no se tratan a tiempo.

Durante la realización de las actividades se buscó empoderar a cada uno de los padres acerca de la salud bucal de sus hijos para así lograr un refuerzo positivo en el acompañamiento diario de su salud oral y, por ende, la disminución del consumo del biberón. Esto se apoya en el estudio de González, Cano, Meneses y Vivares (2014), en el cual se manifestó que las madres refirieron estar dispuestas a

62 brindar buena atención a la salud bucal de sus hijos porque son conscientes de que es su responsabilidad, pero que necesitan acciones educativas por parte del personal de salud y tener más colaboración eficiente de las agentes educativas diariamente dentro del hogar comunitario.

En la siguiente visita se abordaron temas como las causas y las consecuencias del uso prolongado del biberón y las buenas técnicas de higiene oral. En los talleres pedagógicos se pudo evidenciar la actitud asertiva de los padres y las agentes educativas, quienes estuvieron dispuestos a aprender acerca del hábito del biberón, reflejando con esto interés, agrado y complacencia. De acuerdo con esto, es importante resaltar algunas conclusiones obtenidas en un estudio realizado por Muñoz y Mattos (2015), en este los participantes tuvieron actitud positiva hacia el desarrollo de los programas y actividades de promoción de la salud y prevención de la enfermedad, lo que les permitió la construcción de aprendizajes significativos.

En la fase de evaluación con los niños se realizó la actividad "Enterrando recuerdos", en la que por iniciativa propia cada niño decidió enterrar su tetero y explicar la razón. Los niños y niñas refirieron que también estaban enterrando "los monstruos del tetero", además de que ellos eran súper odontólogos y debían acabarlos; estas actitudes denotaron cambios con la transición del biberón al vaso para lograr un beneficio a su salud bucal. Tal como lo explican Mora et al. (2017), los seres humanos somos "animales de hábitos", por ende, las niñas y niños del mismo modo que estuvieron habituados a consumir biberón se pueden acostumbrar a tomar bebidas en el vaso.

En la fase de evaluación se realizó como última actividad con los padres y las agentes educativas un grupo focal final con las mismas preguntas iniciales, con las que posteriormente se constató la disminución del consumo del biberón. Con esta investigación se logró que la mitad de la población dejara el hábito y que los demás participantes redujeran la frecuencia de su uso, pasando de tres o cuatro veces al día a una o dos veces máximo.

Este significativo logro fue posible gracias a la participación activa de las niñas y los niños en las actividades y también al acompañamiento constante de los tutores y agentes educativas que hicieron refuerzos positivos en cada casa de familia y en el hogar comunitario correspondiente. Lo anterior demuestra la importancia de involucrar a todos los actores a cargo de la primera infancia para eliminar o implementar hábitos bucales de manera eficaz. Al menos así lo atestiguan Gaeta, Cavazos y Cabrera "Los padres de familia son un factor de influencia determinante en la adquisición y eliminación de hábitos bucales en sus hijos" (2017, p. 976).

La participación de agentes educativas y tutores fue necesaria, ya que la falta de conocimientos en cuanto a salud oral de la primera infancia tiene influencia en el estado de salud bucal de sus hijos. Por esta razón se debe educar tempranamente en la importancia de dejar el hábito y sus posibles repercusiones negativas en la salud oral de los niños (Delgado, Bravo y Chusino, 2016).

En tal virtud, la presente investigación demostró el aumento del conocimiento acerca del hábito del biberón y prácticas en higiene oral tanto en los padres como las agentes educativas. Las siguientes respuestas dan muestra de lo anterior:

"El tetero se debe tomar hasta los 2 años como un complemento porque si lo deja más tiempo se vuelve un hábito". (5G1) 
"El tetero da malformaciones de la cara, dolor, exodoncias, ortodoncias a futuro y la caries". (7G2)

"La caries es la enfermedad que le da al diente y lo va destruyendo" (2G1)

"Es importante cepillarlos antes de dormir porque de noche es cuando uno no produce saliva y eso ayuda a la caries". (3G2)

Lee, Lee, Ballart, Simón y Guerrero (2015) evidenciaron el aumento del nivel de conocimiento de los padres posterior a la realización de una propuesta que redundara en educación para la salud (por medio de talleres pedagógicos que incluyeran conferencias y actividades pedagógicas), con el posterior abandono de hábitos deformantes de la salud bucodental. Igualmente, la presente investigación ratifica que la educación para la salud, por medio de la PP, logra cambios importantes en la población, ya que no solo se trata de impartir enseñanza sistematizada, sino de permitirles a los participantes construir aprendizaje significativo que les motive a mejorar su calidad de vida.

\section{Conclusiones}

El desconocimiento sobre el hábito del biberón fue alto en la población sujeto; es posible considerar esto como un factor de riesgo para la CIT y las alteraciones en la cavidad oral. Por ello, es prioritario implementar programas específicos de educación para la salud que logren hábitos de vida saludables desde edades tempranas.

En este estudio se logró, mediante la PP, crear un cambio favorable en el bienestar general y bucal de la comunidad mediante su participación en cada una de las actividades que partieron de sus conocimientos previos para así alcanzar un aprendizaje valioso que puedan aplicar en sus vidas.

Así entonces, se logró mediante la educación para la salud y la PP empoderar a la comunidad acerca de su estado de salud bucal, esto con la realización de actividades que no solo se dedicaron a la trasmisión de información sino a la participación de la comunidad en talleres lúdicos que buscaron cambiar de manera significativa los estilos de vida individuales, convirtiéndose así en una herramienta para la promoción de la salud.
Finalmente, eliminar o disminuir un hábito en primera infancia implica, además de trabajar con la persona que lo tiene interiorizado, tener en cuenta su contexto familiar y social. Por ello, se recomienda abordar hábitos bucales en niños y niñas considerando los determinantes sociales e involucrar a los actores responsables de su educación, cuidado y crianza.

\section{Referencias}

Acevedo, O., Mora, C., Capote, J. L., Rosell, C. R. y Hernández, Y. (2011). Efectividad de una intervención educativa en escolares de 8 a 11 años portadores de hábitos bucales deformantes. Medisur, 9(2). Recuperado de http:// scielo.sld.cu/scielo.php?script=sci arttext\&pi$\mathrm{d}=\mathrm{S} 1727-897 \mathrm{X} 2011000200006$

Aguilar, F. J., Duarte, C. G., Rejón, M. E., Serrano, R. y Pinzón, A. L. (2014). Prevalencia de caries de la infancia temprana y factores de riesgo asociados. Acta Pediátrica de México, 35(4), 259-266. Recuperado de http://ojs.actapediatrica.org.mx/index.php/APM/article/ viewFile/785/706 https://doi.org/10.18233/ APM35No4pp259-266

Ávila, P. A., Leiva, L. F., Bueno, J. C. y Gámez, M. F. (2016). Estrategia de educación en salud y ambientes saludables, para niñas, niños y adolescentes bajo protección. Movimiento Científico, 10(2), 41-55. Recuperado de http://revistas.iberoamericana.edu.co/index.php/Rmcientifico/ article/view/1229/933

Carlos, L. E., Luengo, J. A., Toscano, I., Luévano, A. P. y Anaya, M. (2017). Knowledge, beliefs, and perceptions of Mexican university students on early childhood cavities. Acta Universitaria, 27(2), 46-52. https://doi.org/10.15174/ au. 2017.1330

Castro, G. E. (2017). Salud bucal individual y colectiva del niño y del adolescente: una experiencia pedagógica en la Universidad Nacional de Colombia. Acta Odontológica Colombiana, 7(1), 101-119. Recuperado de https://revistas. unal.edu.co/index.php/actaodontocol/article/ view/64078/59396

Chen, X., Xia, B. y Ge, L. (2015). Effects of breast-feeding duration, bottle-feeding duration and 
non-nutritive sucking habits on the occlusal characteristics of primary dentition. BMC Pediatrics, 15(1), 46. Recuperado de https://bmcpediatr.biomedcentral.com/articles/10.1186/ s12887-015-0364-1 https://doi.org/10.1186/ s12887-015-0364-1

Contreras, A. (2016). La promoción de la salud general y la salud oral: una estrategia conjunta. Revista clínica de periodoncia, implantología y rehabilitación oral, 9(2), 193-202. Recuperado de https://www.sciencedirect.com/science/ article/pii/S0718539116300350 https://doi.org/10.1016/j.piro.2016.07.003

Corrales, A. L., Duque, Y., Serrano, D., González, M. y Alonso, A. (2014). La sugestión en una etapa del sueño para el tratamiento de hábitos bucales deformantes. Revista de Ciencias Médicas de Pinar del Río, 18(6), 997-1007. Recuperado de http://scielo.sld.cu/pdf/rpr/v18n6/ rpr07614.pdf

De Luca, E., Rivera, A. y Romero, Y. (2015). Intervención para la promoción de salud bucal dirigido a niños de educación inicial de la Unidad Educativa La Vega de Tabay, municipio Santos Marquina, Mérida, Venezuela. Revista Venezolana de Investigación Odontológica, 3(2), 94110. Recuperado de http://erevistas.saber.ula. ve/index.php/rvio/article/view/6964/6837

Delgado, M. E., Bravo, D. y Chusino, E. (2016). Nivel de conocimiento de higiene oral de las madres y su influencia en la salud bucodental menores 0-3 años, atendidos Centro de salud Jaramijo, marzo-agosto 2014. Publicando, 3(7), 90-97. Recuperado de https://www.rmlconsultores.com/revista/index.php/crv/article/ view/111/pdf 118

Gaeta, M. L., Cavazos, J. y Cabrera, M. del R. L. (2017). Habilidades autorregulatorias e higiene bucal infantil con el apoyo de los padres. Revista Latinoamericana de Ciencias Sociales, Niñez y Juventud, 15 (2), 965-978. DOI:10.11600/1692715x.1521109.

García, L. C., Sánchez, L. A. y Estrada, J. H. (2015). Determinantes estructurales y su relación con el índice de COP en países de desarrollo alto, mediano y bajo. Acta Odontológica Colombiana, 5(1), 25-40. Recuperado de https://revistas. unal.edu.co/index.php/actaodontocol/article/ view/52277/52021

Gil, A. (2017). Mediana de duración de la lactancia materna y prácticas de alimentación complementaria del lactante y niño pequeño menor de 3 años del municipio de La Vega, Cundinamarca. Julio a noviembre de 2016. Informe de resultados. Recuperado de http://www.lavega-cundinamarca.gov.co/Transparencia/Informes/Anexo $\% 20 \mathrm{No} \% 204 . \% 20$ Informe $\% 20$ Mediana\%20de\%20duraci\%C3\%B3n\%20 de\%20la \%20lactancia $\% 20$ materna $\% 20 \mathrm{e} \% 20$ indicadores $\% 20$ OMS- $\% 20$ La $\% 20$ Vega $\% 20$ 2016.pdf

Gómez, A. M., Bernal, T., Posada, A. y Agudelo, A. A. (2015). Caries dental, higiene bucal y necesidades de tratamiento en población de 3 a 5 años de una institución educativa de MedeIlín y sus factores relacionados. Revista Nacional de Odontología, 11(21), 23-35. https://doi. org/10.16925/od.v11i21.933

González, C., Cano, M., Meneses, E. J. y Saldarriaga, V. (2016). Conocimientos en salud bucal de los cuidadores del programa Buen Comienzo. Gerencia y Políticas de Salud, 15(31), 130-144. https://doi. org/10.11600/1692715x.13211270314

González, C., Cano, M., Meneses, E. J. y Vivares, A. M. (2014). Percepciones en salud bucal de los niños y niñas. Revista Latinoamericana de Ciencias Sociales, Niñez y Juventud, 13(2), 715-724. https://doi.org/10.1016/j.anpedi.2014.01.014

González, E., Pérez, S., Alarcón, J. A. y Peñalver, M. A. (2015). Knowledge of Andalusian pediatricians and parents about early-onset tooth decay. Anales de Pediatría, 82(1), 19-26. DOI: 10.1016/j.anpedi.2014.01.014.

Instituto Colombiano de Bienestar Familiar (ICBF) (2014). Lineamientos técnico administrativo y operativo hogares comunitarios en todas sus formas (fami, familiares, grupales, múltiples, múltiples empresariales y jardines sociales) para la atención a los niños y niñas hasta los cinco (5) años de edad. Recuperado de https://www.icbf.gov.co/sites/default/ files/anexo 6. lineamiento hogares comunitarios.pdf 
Jiménez, A., Galvis, J., Gómez, V., Salinas, A. y Agudelo, A. (2015). Proyecto pedagógico para el aprendizaje de prácticas bucales saludables con niños y niñas escolarizados entre 8 y 10 años. Infancias Imágenes, 14(1), 40-49. Recuperado de https://revistas.udistrital.edu.co/ojs/ index.php/infancias/article/view/8232/10427 https://doi.org/10.14483//udistrital.jour. infimg.2015.1.a03

Jiménez, J. (2016). Importancia de la deglución atípica en las maloclusiones. Odontología Sanmarquina, 19(2), 41-44. https://doi. org/10.15381/os.v19i2.12917

Lee, Y., Lee, Y., Ballart, A., Simón, J. y Guerrero, I. (2015). Intervención educativa sobre hábitos bucales deformantes en escolares de 6 a 12 años. Revista de Información Científica, 93(5), 1121-1131. Recuperado de http://www. revinfcientifica.sld.cu/index.php/ric/article/ view/182/1400

Ministerio de Salud y Protección Social (2014). IV estudio nacional de salud bucal-Ensab IV. Recuperado de https://www.minsalud.gov.co/ sites/rid/Lists/BibliotecaDigital/RIDE/VS/PP/EN$\underline{\text { SAB-IV-Situacion-Bucal-Actual.pdf }}$

Ministerio de Salud (1999). III estudio nacional de salud bucal-Ensab III. Bogotá: Ministerio de Salud.

Mora, C., Álvarez, I., Blanco, A., Espino, Y., Morera, A. y Macías, A. (2017). Efectividad de la intervención en niños de 5 a 11 años portadores de hábitos bucales deformantes. Revista Nacional de Odontología, 13(25), 1-20. Recuperado de https://revistas.ucc.edu.co/index.php/od/ article/view/1884/1975

Muñoz, W. E. y Mattos, M. A. (2015). Técnica educativa para mejorar los conocimientos de salud oral de madres y reducir el índice de higiene oral de preescolares. International Journal of Odontostomatology, 9(2), 321-327. Recuperado de https://scielo.conicyt.cl/pdf/ijodontos/v9n2/art21.pdf https://doi.org/10.4067/ $\underline{\text { S0718-381X2015000200021 }}$

Myers, D. G. (2005). Psicología. Bogotá: Editorial Médica Panamericana.

Nihi, V. S., Maciel, S. M., Jarrus, M. E., Nihi, F. M., Salles, C. L. F., Pascotto, R. C. y Fujimaki, M. (2015). Pacifier-sucking habit duration and frequency on occlusal and myofunctional alterations in preschool children. Brazilian Oral Research, 29(1), 00-13. Recuperado de http:// www.scielo.br/scielo.php?script=sci arttextypid=S1806-83242015000100211 https://doi.org/10.1590/1807-3107BOR-2015.vol29.0013

Ocampo, A., Johnson, N. y Lema, M. C. (2013). Hábitos orales comunes: revisión de literatura. Parte I. Revista Nacional de Odontología, 9, 8390. Recuperado de https://revistas.ucc.edu.co/ index.php/od/article/view/434/435

Organización de las Naciones Unidas para la Educación, la Ciencia y la Cultura (Unesco) (1999). $6 b$ Promoción y educación para la salud. Recuperado de http://www.unesco.org/education/ uie/confintea/pdf/6b span.pdf

Peñaranda, F., López, J. M. y Molina, D.P. (2017). La educación para la salud en la salud pública: un análisis pedagógico. Hacia la Promoción de la Salud, 22(1), 123-133. https://doi. org/10.17151/hpsal.2017.22.1.10

Reni, K. y Piñeiro S. (2014). Malos hábitos orales: rehabilitación neuromuscular y crecimiento facial. Revista Médica Clínica Condes, 25(2), 380-388. Recuperado de https://www. sciencedirect.com/science/article/pii/ S0716864014700501 https://doi.org/10.1016/ S0716-8640(14)70050-1

Reyes, D. E., Torres, I., Quesada, L. M., Milá, M. y Labrada, H. E. (2014). Hábitos bucales deformantes en niños de 5 a 11 años. Medisan, 18(5), 606-612. Recuperado de http:// scielo.sld.cu/scielo.php?script=sci arttextypi$\mathrm{d}=\mathrm{S} 1029-30192014000500003$

Rincón, G. (2012). Los proyectos de aula y la enseñanza y el aprendizaje del lenguaje escrito. Bogotá: Kimpres.

Salamanca, R. y Murrieta, J. F. (2015). Frecuencia de alteraciones en la oclusión en dentición primaria y su asociación con algunos hábitos bucales parafuncionales en un jardín de niños del estado de México. Revista Científica Odontológica, 11(2), 8-15. Recuperado de http://www.redalyc. org/html/3242/324243574002/index.html

Sánchez, M. K., Sánchez, K. J. y Agudelo, A. (2015). Estrategias lúdicas para aumentar el conocimiento de un grupo de adolescentes escolarizados 
sobre la gingivitis. Duazary: Revista Internacional de Ciencias de la Salud, 12(2), 100-111. Recuperado de http://revistas.unimagdalena.edu. co/index.php/duazary/article/view/1466/854 https://doi.org/10.21676/2389783X.1466

Secretaría de Salud de Risaralda (2017). Situación problema. Recuperado de http://www.risaralda. gov.co/salud/publicaciones.php?id=100887

Secretaría de Salud y Seguridad Social de Pereira (2014). Análisis de situación de salud con el modelo de los determinantes sociales de salud (Asis). Recuperado de http://www.pereira.gov. co/Transparencia/PlaneacionGestionyControl/ An\%C3\%A1lisis\%20de\%20la\%20Situaci\%C3\%B3n\%20de\%20Salud\%202014.pdf

Tello, G., Abanto, J., Butini, L. B., Murakami, C. M., Bonini, G. y Bönecker, M. (2016). Impacto de los principales problemas de salud bucal en la calidad de vida de preescolares. Odontología, 19(2), 42-52. Recuperado de http://revistadigital.uce.edu.ec/index.php/ODONTOLOGIA/
Torres, G., Blanco, D. J., Chávez, M. G., Apaza, S. y Antezana, V. (2015). Impacto de la caries de la infancia temprana en la calidad de vida relacionada a la salud bucal en niños peruanos menores de 71 meses de edad. Odontología Sanmarquina, 18(2), 87-94. Recuperado de http://revistasinvestigacion.unmsm.edu.pe/ index.php/odont/article/view/11520/10358 https://doi.org/10.15381/os.v18i2.11520

Universidad Nacional de Colombia (2016). Guía de atención de mordidas cruzadas. Bogotá: Universidad Nacional de Colombia. Recuperado de http://www.odontologia.unal.edu.co/docs/ habilitacion/guia ort mord_cruz.pdf

Varela, M. y Vives, T. (2016). Autenticidad y calidad en la investigación educativa cualitativa: multivocalidad. Investigación en Educación Médica, 5(19), 191-198. Recuperado de http://www.scielo.org.mx/scielo. php?pid=S2007-50572016000300191\&script=sci arttext https://doi.org/10.1016/j. riem.2016.04.006 